\title{
Transport Behaviour of Selected Nanoparticles with different Surface Coatings in Granular Porous Media coated with Pseudomonas aeruginosa Biofilm
}

\author{
Revised and resubmitted to: \\ Environmental Science and Technology \\ for consideration for the \\ Special Issue on "Transformations of Nanoparticles in the Environment"
}

December 3, 2011

SHWETA TRIPATHI, DENIS CHAMPAGNE, and NATHALIE TUFENKJI*

Department of Chemical Engineering, McGill University,

Montreal, Quebec

H3A 2B2, Canada

${ }^{*}$ Corresponding Author. Phone: (514) 398-2999; Fax: (514) 398-6678; E-mail: nathalie.tufenkji@mcgill.ca 
Abstract

Well-controlled laboratory column experiments were conducted to understand the influence of Pseudomonas aeruginosa ( $P$. aeruginosa) biofilms on the transport of selected engineered nanoparticles (ENPs) in granular porous media representative of groundwater aquifers or riverbank filtration settings. To understand the importance of particle size on retention in the biofilm-coated granular (quartz sand) matrix, column experiments were carried out using nano-sized (20 nm) and micronsized $(1 \mu \mathrm{m})$ sulfate-functionalized polystyrene latex particles (designated as $20 \mathrm{nSL}$ and $1 \mathrm{mSL}$, respectively). Additional experiments conducted with nano-sized $(20 \mathrm{~nm})$ carboxyl-modified latex particles $(20 n \mathrm{CL})$ and carboxyl-modified $\mathrm{CdSe} / \mathrm{ZnS}$ quantum dots (QDs) provide information on the influence of particle surface chemistry on retention. Biofilm grown on the surface of the sand was characterized by total biomass quantification, confocal laser scanning microscopy (CLSM) and electrokinetic analysis. All four particles exhibit increased retention in the biofilm-coated packed bed: e.g., the attachment efficiency $(\alpha)$ of the $1 \mathrm{mSL}$ particle increases from 0.40 to 1.7 , whereas $\alpha$ for the $20 n S L$ particle increases from 0.04 to 0.10 in the biofilm-coated system. Particle surface chemistry can also influence the affinity of the ENPs for the biofilm coating as revealed by the greater attachment of the $20 n$ SL particle onto the biofilm-coated sand $(\alpha=0.10)$ than its carboxylated counterpart $(\alpha=0.04)$. Column experiments conducted using sand coated with growth medium (LB) or extracellular polymeric substances (EPS) extracted from $P$. aeruginosa biofilms further reveal that particle surface chemistry influences the interaction between the different ENPs and these coated sand surfaces. Namely, coating of sand surfaces with LB medium or bacterial EPS does not affect the transport of the sulfonated nanoparticle, but the LB coating leads to decreased retention of the carboxylated latex nanoparticle. Furthermore, our results show that EPS coatings are not necessarily good surrogates for biofilm-coated sand. Electrokinetic characterization of the clean and coated sand surfaces also reveals that the extent of particle retention is not controlled by electrical double layer interactions. Future studies should thus be aimed at improving our understanding of the fundamental mechanisms (both colloidal and non-colloidal) governing nanoparticle transport and fate in biofilm-laden granular aquatic environments. 


\section{Introduction}

Global increases in investment on nanotechnology research and development have led to an exponential rise in commercial applications involving engineered nanoparticles (ENPs) ${ }^{l}$. Nanoscale materials can be found in a variety of products and processes such as electronics, cosmetics, pharmaceuticals, energy and materials applications ${ }^{2}$. The wide scale use of ENPs may result in large quantities of these materials being released in the environment as industrial and domestic wastes ${ }^{2}$. Unfortunately, several ENPs are suspected of posing environmental and/or health risks ${ }^{1,2}$. Some ENPs, when released in the environment, can migrate in the subsurface where they may interact with soil organisms, and contaminate groundwater supplies which are an important source of potable water. It is therefore of interest to understand the fate and transport of ENPs in aquatic environments, such as those representative of groundwater aquifers, riverbank filtration settings and engineered water treatment facilities 3,4

Following introduction into natural subsurface environments or engineered water treatment systems (e.g., deep-bed filtration), the transport and fate of ENPs is governed by a complex interplay of particle-surface and particle-particle interactions $3^{3}$. Accordingly, several recent laboratory studies have been aimed at understanding the influence of environmental factors (e.g., water chemistry, flow velocity, collector surface chemistry, size and shape) and ENP properties (e.g., size, shape, and surface chemistry) on their transport and aggregation in granular porous media ${ }^{3}$. However, the natural subsurface environment and engineered treatment facilities are complex heterogeneous systems that may not be well represented by homogenous granular materials such as the clean glass beads or quartz sand commonly used to study ENP transport in packed columns. For instance, biofilms are ubiquitous 
in many natural and engineered environments, yet there is limited information on the influence of these microbial structures on the transport and fate of ENPs in granular aquatic matrices ${ }^{5-9}$.

Biofilms are structured communities of microorganisms enclosed within a self-developed matrix of excreted polymeric substances and adherent to a living or inert surface ${ }^{10}$. Biofilm accumulation in granular porous matrices affects not only the surface properties of the granular media, but can also alter hydraulic conditions such as permeability and flow patterns. Yet, a limited number of studies have examined the role of biofilms in the fate and transport of ENPs in granular systems ${ }^{5-9}$. Leon Morales et al., 9 showed that $P$. aeruginosa SG81 biofilms significantly retarded the transport of $30 \mathrm{~nm}$ laponite clay particles in columns packed with sand when suspended in a $\mathrm{CaCl}_{2}$ solution. In contrast, transport of the laponite particles was enhanced in the biofilm-coated column when the clay was suspended in a monovalent $\mathrm{NaCl}$ solution. Tong et $\mathrm{al} .{ }^{5}$ demonstrated that the presence of $E$. coli biofilms on the surface of quartz sand leads to increased retention of nano-sized fullerene $\left(n \mathrm{C}_{60}\right)$ particles. In their study ${ }^{5}$, the transport of $n \mathrm{C}_{60}$ was retarded when suspended in either $\mathrm{NaCl}$ or $\mathrm{CaCl}_{2}$ solutions; however, the overall effect of the biofilm coating on nanoparticle retention was not dramatic. Interestingly, the measured deposition rate of the $n \mathrm{C}_{60}$ particles in the packed column did not increase by more than $50 \%$ as a result of biofilm coating over the range of water chemistry examined 5 . Peulen and Wilkinson ${ }^{6}$ used CLSM and fluorescence correlation spectroscopy (FCS) to study the diffusion of dextrans, nano-sized carboxylated latex $(n \mathrm{CL})$, and nano-sized silver $(n \mathrm{Ag})$ particles in biofilms of $P$. fluorescens. They observed that the charge of the nanoparticles was an important factor in their diffusion behavior; the negatively charged $n \mathrm{Ag}$ exhibited significant decrease in the self-diffusion coefficient in a dense biofilm. FCS has also been used to investigate the diffusion of $n$ CL in biofilms 
of Lactococcus lactis (L. lactis) ${ }^{8}$. Using genetically modified hydrophilic and hydrophobic mutants of L. lactis, Habimana et $\mathrm{a}^{8}$ showed that bacterial surface hydrophobicity influences the diffusion of $n \mathrm{CL}$ in a biofilm matrix. These few studies clearly suggest that the presence of biofilms can alter the transport behavior of ENPs in natural and engineered aquatic environments; however, our understanding of this process is too limited to allow the development of functional relationships between ENP transport potential and the physical and/or physicochemical properties of ENPs. Little is known about the effect of ENP size and surface properties on their transport in biofilm-coated granular aquatic environments. Moreover, our understanding of the mechanisms that govern ENP transport and fate in biofilm-laden systems is limited.

In this study, we used well-controlled laboratory column experiments to examine the transport behavior of selected ENPs in $P$. aeruginosa biofilm-coated sand. The effect of particle size was investigated by using nano-sized and micron-sized sulfate-functionalized model latex particles. Additional experiments conducted with $n \mathrm{CL}$ and carboxylated quantum dots (QDs) gave further insight on the influence of particle surface chemistry on the transport behavior of the selected ENPs. Columns packed with sand coated with growth medium or bacterial EPS were also used to understand the role of these coatings on ENP transport behavior. Physicochemical characterization of the ENPs and collector surfaces (including biofilm-coated sand) was also undertaken in an effort to better understand the key transport and retention processes in the coated matrices. 


\section{Materials and Methods}

Preparation and Characterization of Particle Suspensions. Table 1 summarizes the properties of the selected particles. Fluorescent sulfonated polystyrene latex beads with nominal diameters of $1 \mu \mathrm{m}$ $(1 \mathrm{mSL})$ and $20 \mathrm{~nm}(20 \mathrm{nSL})$ (Invitrogen), carboxylated polystyrene latex beads with nominal diameter of $20 \mathrm{~nm}(20 n \mathrm{CL})$ (Invitrogen) and carboxylated CdSe/ZnS QDs (Ocean Nanotech) with nominal diameter of $10 \mathrm{~nm}$ were used. Suspensions having the desired particle concentrations were prepared by diluting stocks in filtered $(0.2 \mu \mathrm{m}$ nylon filter, Fisher) $10 \mathrm{mM} \mathrm{KCl}$ solution at $\mathrm{pH} 7.2$ to obtain suspensions at a concentration of $8 \times 10^{11}$ particles $/ \mathrm{mL}$ for the 3 ENPs, and $10^{7}$ particles $/ \mathrm{mL}$ for the micron-sized particle.

\section{[TABLE_1_HERE]}

The hydrodynamic diameters of the ENPs were characterized using dynamic light scattering (DLS) (Zetasizer Nano, Malvern). Laser Doppler Velocimetry in conjunction with Phase Analysis Light Scattering (Zetasizer Nano, Malvern) was used to measure the electrophoretic mobility (EPM) of the ENPs. EPMs were converted to zeta $(\zeta)$ potential using the Smoluchowski equation ${ }^{11}$ for the larger $1 \mathrm{mSL}$ particle, and using the approach reported by Ohshima for the three ENPs ${ }^{12}$. Each measurement was done in triplicate and repeated with four different samples.

Preparation of Biofilm-Coated and EPS-Coated Sand. High purity quartz sand (US mesh size 20/+25 fraction, Granusil \#2040, Ottawa plant, Unimin) was selected as the granular material to allow careful control of the experimental system. The sand was sterilized by autoclaving for $25 \mathrm{~min}$ at $121^{\circ} \mathrm{C}$ and then soaked overnight in sterile electrolyte $(10 \mathrm{mM} \mathrm{KCl}, \mathrm{pH} 7.2)$ prior to packing into the sterile 
glass column as described previously ${ }^{13}$. For biofilm-coated columns, autoclaved sand was soaked in diluted Luria-Bertani (LB) Lennox broth (4 g/L, Fisher) and stored overnight in a non-shaking incubator at $37^{\circ} \mathrm{C}$, prior to packing the column. The Gram-negative rod-shaped, aerobic, EPSproducing bacterium $P$. aeruginosa ATCC 27853 was used for biofilm formation. A pure culture of $P$. aeruginosa was maintained at $-80^{\circ} \mathrm{C}$ in LB broth $(20 \mathrm{~g} / \mathrm{L}$, Fisher) with $15 \%$ glycerol. One week prior to inoculation, the frozen culture was streaked onto a LB agar plate that was incubated at $37^{\circ} \mathrm{C}$ for 21 h. For each biofilm column experiment, a pre-culture was prepared by inoculating $150 \mathrm{~mL}$ of sterile LB broth (in a $500 \mathrm{~mL}$ baffled flask) using a single colony from the LB plate. This culture was incubated at $37^{\circ} \mathrm{C}$ and $200 \mathrm{rpm}$ for $21 \mathrm{~h}$. After $21 \mathrm{~h}, 1 \mathrm{~mL}$ of this pre-culture was used to inoculate 1.6 L of diluted LB broth $(4 \mathrm{~g} / \mathrm{L})$ that was then injected into the media-coated sand column (at 0.8 $\mathrm{mL} / \mathrm{min}$ for $24 \mathrm{hrs}, 37^{\circ} \mathrm{C}$ ) for development of biofilm in the column. Following biofilm formation in the column, a $10 \mathrm{mM} \mathrm{KCl}$ solution was pumped through the column at $4 \mathrm{~mL} / \mathrm{min}$ for $20 \mathrm{~min}$ to flush loose biomass from the pore space. The biofilm-coated sand packed column was then used for particle transport experiments. Control experiments were conducted using sterile columns packed with LBcoated sand. Regular examination of column effluent did not show any evidence of microbial contamination.

To understand the role of biofilm EPS on particle transport, column studies were also conducted with EPS-coated sand. EPS has been reported as the key component of biofilm that can determine the physicochemical and biological properties of biofilms ${ }^{14,15}$. EPS was extracted from $P$. aeruginosa biofilms using the rapid centrifugation method $^{16}$. Briefly, biofilms grown on glass wool were extracted in $10 \mathrm{mM}$ HEPES Buffer (Sigma) by sonication at $40 \mathrm{kHz}$ for 2 min (FS60H bath 
sonicator, Fisher). Formaldehyde (37\%) (Fisher) was added to the extracted biofilm and the solution was stored at $4^{\circ} \mathrm{C}$ for $1 \mathrm{hr}$. This was followed by addition of $1 \mathrm{~N} \mathrm{NaOH}$ and storage in $4^{\circ} \mathrm{C}$ for an additional $4 \mathrm{hrs}$. After refrigerating for $4 \mathrm{hrs}$, the biofilm extract was centrifuged at $20,000 \mathrm{~g}, 4^{\circ} \mathrm{C}$ for 20 min. The supernatant was subjected to dialysis in water (3500 Da dialysis membrane, Fisher) for 24 hrs to obtain the EPS extracted from biofilm in the solution. Autoclaved sand was soaked in this EPS solution overnight in a static incubator at $37^{\circ} \mathrm{C}$ to obtain the EPS-coated sand that was then used to wet pack the column for transport studies.

Characterization of Granular Material. Streaming potential analysis (Electrokinetic Analyzer, Anton Paar) was used to characterize the electrokinetic properties of the sand surfaces. Clean, biofilm-coated, EPS-coated, or LB-coated sand was carefully packed into a cylindrical cell (Anton Paar) and streaming potential was measured by pumping a solution of $10 \mathrm{mM} \mathrm{KCl}(\mathrm{pH} 7.2)$ across the packed cell as described previously ${ }^{17}$. The Fairbrother-Mastin equation ${ }^{18}$ was used to convert measured streaming potentials to zeta potentials (VisioLab for EKA 1.03, Anton Paar). For the biofilm-coated sand, the presence of biofilm on the sand surface before and after streaming potential measurements was confirmed by staining the sand with the nucleic acid stain SYTO9 (Invitrogen) and imaging the biofilm by fluorescence microscopy (EX:490 nm, EM:520 nm, Olympus IX71).

Confocal laser scanning microscopy (CLSM, Zeiss LSM 510 META) equipped with argon laser $(488 \mathrm{~nm})$ and BP 505-530 filter was used to determine the approximate thickness of biofilm formed on the sand surface. Biofilm-coated sand was carefully extruded from packed columns into four equal sections. Twenty-five random sand grain samples were selected from each section, stained 
with SYTO9, and observed at 10× magnification by CLSM. A cross-sectional image of each sand grain was recorded using ZEN 2009 LE software and analyzed using image processing software (Image J, NIH). Biofilm thickness for each sand grain was determined by averaging 20 thickness measurements evenly distributed around the perimeter of the grain.

Column Transport Experiments. Transport experiments were performed using glass columns (8 $\mathrm{cm}$ packed length, $1.6 \mathrm{~cm}$ internal diameter) wet packed with the following types of media, as described above: (i) clean sand, (ii) LB-coated sand, or (iii) EPS-coated sand. Some of the LB-coated sand columns were used for biofilm development after packing as described above. Columns packed with clean or coated sand were fully saturated and equilibrated with $10 \mathrm{mM} \mathrm{KCl}(\mathrm{pH}$ 7.2) for 2 hrs at $0.8 \mathrm{~mL} / \mathrm{min}$. A particle suspension prepared in $10 \mathrm{mM} \mathrm{KCl} \mathrm{(pH} \mathrm{7.2)} \mathrm{was} \mathrm{then} \mathrm{injected} \mathrm{into} \mathrm{the}$ column for $20 \mathrm{~min}(\sim 6$ pore volumes (PVs)), followed by particle-free electrolyte for $30 \mathrm{~min}$. Effluent particle concentration was continuously monitored using a fluorospectrophotometer (Fluoromax 4, Horiba Jobin Yvon) and a 1-cm flow-through cell. The concentration of the influent particle suspension $\left(C_{0}\right)$ was the same as that used for characterization of the particles. Each particle transport experiment was repeated at least twice. Tracer experiments were conducted using $10 \mathrm{mM} \mathrm{KNO}_{3}$ in each of the four treatments. In the case of biofilm-coated, LB-coated, and EPS-coated sand experiments, the column was sliced into four equal sections after each experiment. These sections were then dried at $120^{\circ} \mathrm{C}$ for $14 \mathrm{hrs}$ and the weight of dried sand measured. Following this, the sections were subjected to $800^{\circ} \mathrm{C}$ heating for 24 hrs to burn the organic mass, and re-weighed. Total biomass in each column section was determined from the loss of combusted mass. 


\section{Results and Discussion}

Particle Characterization. The average hydrodynamic diameter of the three different ENPs and the micron-sized particle was determined by DLS at the conditions used in the column transport experiments $(10 \mathrm{mM} \mathrm{KCl}, \mathrm{pH}$ 7.2). The measured sizes of the sulfonated and carboxylated latex particles are relatively close to their commercially reported values (Table 1); however, the QD shows a marked difference between its reported $(10 \mathrm{~nm})$ and DLS-measured $(108 \mathrm{~nm})$ size. Because Rayleigh scattering is proportional to the particle diameter raised to the $6^{\text {th }}$ power, the DLS-measured particle sizes are biased by the largest aggregates ${ }^{19}$. Hence, the DLS measurements suggest that the QD has a tendency to aggregate in the experimental solution. This observation is further supported by measurements conducted using nanoparticle tracking analysis (NTA) that reveal the polydispersity of the QD suspension in $10 \mathrm{mM} \mathrm{KCl} \mathrm{(Supporting} \mathrm{Information,} \mathrm{Figure} \mathrm{S1).} \mathrm{Interestingly,} \mathrm{transmission}$ electron microscopy (TEM) analysis of QDs suspended in low ionic strength electrolyte similar to the manufacturer's stock solution $(0.1 \mathrm{mM} \mathrm{KCl}, \mathrm{pH} 7)$ does not reveal significant aggregation of the stock QD suspension (Supporting Information, Figure S2).

The surface charge of ENPs has been shown to play an important role in ENP transport and deposition onto sand grains and model sand surfaces ${ }^{20-24}$. Thus, the $\zeta$-potential of the particles was determined under the conditions used in the column experiments and found to be negative for all of the particles (Table 1). The $1 \mathrm{mSL}$ particles are most negatively charged followed by the $20 n \mathrm{SL}, 20 n \mathrm{CL}$ and the QD in decreasing order of their absolute surface potentials. Because $\zeta$-potential can be an 
important parameter governing colloid stability, its role in the observed ENP transport behavior will be discussed in a later section.

Characterization of Collector Surfaces. Figure 1 shows a comparison of the $\zeta$-potentials for the different collector surfaces used in this study. The $\zeta$-potential of clean sand is negative $(-15.3 \pm 0.6$ $\mathrm{mV}$ ) under the experimental conditions. All three organic coatings (biofilm, EPS, and LB) decrease the absolute surface potential of the clean sand, whereby biofilm-coated $(-8.5 \pm 0.3 \mathrm{mV})$ and EPScoated sand $(-9.1 \pm 0.5 \mathrm{mV})$ exhibit comparable $\zeta$-potentials, suggesting a similarity between the two types of coatings with respect to charged functional groups. Although the $\zeta$-potential of collector surfaces is known to play an important role in determining particle-surface interactions in aquatic systems ${ }^{3}$, the surface potential of biofilm-coated collectors has not been previously reported in this context. Truesdail et al. ${ }^{17}$ showed that positively charged aluminum hydroxide-coated sand continuously exposed to wastewater becomes more negatively charged due to surface conditioning and biofilm formation on the surface. The data in Figure 1 further demonstrate that streaming potential analysis is a useful technique for characterizing the electrokinetic properties of biofilm-coated collector surfaces in aquatic media.

[FIGURE_1_HERE]

Collector surfaces were further characterized by measuring the total attached biomass (biofilm, EPS, and LB components) per unit mass of sand for the packed column. The total biomass for the biofilmcoated columns $(3.11 \pm 0.35 \mathrm{mg} / \mathrm{g}$ sand) is much higher than the total biomass on the surface of LB- 
coated $(0.71 \pm 0.09 \mathrm{mg} / \mathrm{g}$ sand $)$ and EPS-coated sand columns $(0.89 \pm 0.05 \mathrm{mg} / \mathrm{g}$ sand $)$. These measurements confirm the presence of organic coatings on the collector surfaces and establish the reproducibility of the coating procedure. Biomass measurements were also used to establish the longitudinal profile of biofilm growth within the packed columns (Figure 2a). Significantly more biomass is observed at the influent end (top) of the column as compared to the effluent end. This observation can be attributed to several reasons, including (i) classical physicochemical filtration (as well as physical straining) inherently results in a decay in the number of retained cells with transport distance; and (ii) higher availability of nutrients at the column influent.

[FIGURE_2_HERE]

CLSM combined with fluorescent staining was used to quantify the approximate thickness of biofilm formed on the surface of the sand grains (Figure 2b). The average biofilm thickness is $22 \pm 6 \nabla \mathrm{m}$ whereas the mean equivalent spherical diameter of the sand grains is approximately $0.76 \mathrm{~mm}$. Thus, biofilm coating increased the effective diameter of the collectors only by $\sim 5 \%$. Imaging of sand grains sampled from different locations in the column indicates that biofilm thickness is similar along the entire length of the packed column (data not shown). Hence, the observation of variable biomass but similar biofilm thickness along the length of the column suggests that biomass (possibly sloughed from the biofilm) is present in the interstices between the collector grains towards the top of the column.

Transport and Retention of Selected ENPs in LB-, Biofilm-, and EPS-Coated Sand. Prior to injecting ENPs into the sand packed columns, the breakthrough behavior of an inert tracer was 
examined (Figure S3). Tracer breakthrough curves (BTCs) for the biofilm-coated columns showed an early appearance of a small amount of tracer (Fig. S3c). However, the bulk of the tracer appeared after a duration of approximately 7 min which is similar to the breakthrough time of the ENPs through the columns irrespective of the collector surface (Figure S3 and Table S1). Previous studies have reported that biofilm formation can cause bioclogging leading to changes in system hydrodynamics ${ }^{25}$, however, such a dramatic effect was not observed in our system. The relatively high influent flow rate and extensive column equilibration may explain the unchanged hydrodynamics of the system in spite of biofilm formation.

Colloid filtration theory (CFT) was used to interpret the results of column experiments for the different ENPs and treatments. The attachment efficiency $(\mathbb{\nabla})$ in CFT is a useful parameter to compare the transport and deposition behavior of different particles under varying environmental conditions. ENP attachment efficiencies were calculated from each measured particle BTC as follows:

$$
\alpha=-\frac{2}{3} \frac{d_{50}}{(1-\varepsilon) L \eta_{0}} \ln \left(\frac{C}{C_{0}}\right)
$$

where $d_{50}$ is the average diameter of the sand grains, $\bigotimes$ is the bed porosity, $L$ is the packed-bed length, and $\nabla_{\Delta} \nabla_{i s}$ the theoretical single-collector contact efficiency evaluated using a correlation equation developed by Tufenkji and Elimelech ${ }^{26}$. The value of $C / C_{0}$ in eq. 1 is obtained from the BTCs recorded from the particle transport experiments.

Figure 3 shows a comparison of the measured attachment efficiency for the micron-sized particle (1mSL) and the three ENPs (20nSL, 20nCL, and QD) when introduced into clean or coated sand-packed columns. In the clean sand columns, the largest particle $(1 \mathrm{mSL})$ is retained to a much 
greater extent $(\mathbb{\otimes}=0.40)$ than the smaller ENPs (average $\bigotimes=0.05$ ), even though $1 \mathrm{mSL}$ exhibits the most negative $\zeta$-potential. As discussed above, the clean sand surface is negatively charged under the conditions of the transport experiment (Figure 1). Hence, the deposition of the negatively charged $1 m S L$ particle onto the clean sand is considered to be unfavorable; namely, $1 \mathrm{mSL}$ experiences repulsive electrical double-layer interactions as it approaches the sand surface. Particle-surface interaction energy profiles were calculated using the classical Derjaguin-Landau-Verwey-Overbeek (DLVO) theory which describes the interaction potential between charged surfaces as the sum of electrical double layer (EDL) and van der Waals (VDW) interactions ${ }^{27}$, 28. Although DLVO calculations have certain inherent limitations (e.g., they do not account for the heterogeneity of the coated sand surfaces), these calculations can be used to estimate the height of the repulsive energy barrier $\left(\Phi_{\max }\right)$ and the depth of the secondary energy well $\left(\Phi_{2^{\circ}}{ }^{\circ}\right)$ upon approach of the negatively charged particles to the negatively charged clean sand surface. In this study, the total interaction energy, namely, the sum of VDW and EDL interactions, was determined by treating the particle-sand system as a sphere-plate interaction. EDL interactions were calculated using the linear superposition approximation of Gregory ${ }^{29}$, where the $\zeta$-potentials of the particles (Table 1) and the clean sand surface (Figure 1) were used in place of the respective surface potentials. The retarded VDW attractive interaction energy was calculated from the expression proposed by Gregory ${ }^{30}$. Values of $1.0 \times 10^{-20} \mathrm{~J}$ and $1.02 \times 10^{-20} \mathrm{~J}$ were chosen for the Hamaker constant for the silica-water-latex and the silica-water-QD systems, respectively ${ }^{3}$. Particle sizes were taken from values obtained from the DLS measurements (Table 1).

Table 2 summarizes the key parameter values obtained from particle-surface interaction energy calculations based on the classical DLVO theory. The predicted depth of the secondary energy well 
$\left(\Phi_{2^{\circ} \mathrm{min}}\right)$ is relatively small indicating that retention in the secondary minimum is expected to be negligible for the three ENPs. DLVO calculations predict a repulsive energy barrier of $\sim 180 k T$ as the $1 m S L$ particle approaches the clean sand surface (where $k$ is the Boltzmann constant and $T$ is the absolute temperature). The three smaller ENPs (20nSL, 20nCL, and QD) are also repelled by the clean sand surface, but the predicted height of the repulsive energy barrier $\left(\Phi_{\max }\right)$ is much lower for these particles (Table 2). Thus, based on consideration of particle retention in the primary energy well alone (classical interpretation of particle deposition), the three smaller ENPs are expected to deposit to a much greater extent than the larger $1 \mathrm{mSL}$ particle. The noted disagreement between the experimental observations and the predictions based on the classical interpretation of particle deposition in the primary energy well suggest that the retention of $1 \mathrm{mSL}$ particles in the sand column may not be solely governed by this simple physicochemical filtration process. Table 1 shows that the $1 m S L$ particle experiences an attractive interaction on the order of $1 k T$ as it approaches the clean sand surface. Hence, it is also possible that this particle experiences some retention in the secondary energy well, which would explain the greater extent of retention of the $1 \mathrm{mSL}$ particle in the clean sand system 31,32. Furthermore, a physical mechanism such as straining may also be contributing to the removal of $1 m S L$ particles from the pore fluid ${ }^{33}, 34$. The measured hydrodynamic diameter of the QD is much greater than that of the polystyrene latex nanospheres, and this ENP is expected to experience more repulsion as it approaches the clean sand surface (Table 2), yet it exhibits a greater attachment efficiency than the latex nanospheres in the clean sand column (Figure 3). Although the predicted depth of the secondary energy well for the QD is not very significant (Table 2), the larger aggregates present in the QD suspension will experience greater attraction than that predicted by the average 
particle size indicated in Table 1. Hence, some QD aggregates might be retained in the secondary energy well. Straining may also contribute to the removal of QD aggregates, which would explain the greater retention of this ENP onto the clean sand in comparison to the two polystyrene nanoparticles.

[TABLE_2_HERE]

[FIGURE_3_HERE]

The first step in biofilm formation on surfaces is the establishment of a "conditioning film", or layer of adsorbed organic and inorganic molecules from the local surrounding aquatic environment ${ }^{35}, 36$. To better understand how the presence of such a conditioning film might influence ENP transport in saturated granular porous media, experiments were also conducted using sand coated with diluted LB medium. The undefined LB growth medium consists of a complex mixture of peptides, proteins, vitamins, trace elements, and minerals that can adsorb onto and mask the available favorable deposition sites on the sand surface. Yet, the average $\zeta$-potential of the LB-coated sand is less negative than the clean sand, indicating that even if the components of LB mask the favorable patches on the sand and coat the sand surface, the overall effect is not significant enough to yield a more negatively-charged surface. Although DLVO calculations would not be meaningful for this highly heterogeneous system, the different particles are generally expected to experience lessened EDL repulsion upon approach to the LB-coated sand versus the clean sand. Nevertheless, in general, the particles exhibit lower retention in the LB-coated sand column (note: the effect is not significant for the $20 n \mathrm{SL}$ particle). As mentioned above, LB media is a complex mixture of organic and inorganic compounds, and adsorption of these components onto the clean sand can result in a highly heterogeneous surface that may give rise to diverse colloidal and non-colloidal interactions upon 
approach of the different particles. Steric repulsion of nanoparticles, bacteria, or other colloids as they approach polymer-coated surfaces in aquatic media has been reported for different experimental systems ${ }^{37-42}$. Given the diversity of polymers and chemical functionalities present in the undefined growth medium, steric repulsion of the selected micron- and nano-sized particles upon approach to the LB-coated sand surface is very likely, irrespective of particle size and surface chemistry.

The LB-coated sand packed columns were used to establish biofilm-coated matrices for the study of particle transport. In general, $P$. aeruginos a biofilms reduce the transport potential of the selected ENPs in the packed bed (Figure 3). This observation is consistent with some previous studies examining colloid or bacteria transport in biofilm-coated sand packed columns, ${ }^{93-47}$. The overall trend in particle retention for the biofilm-coated sand columns is similar to that observed for the clean sand (and LB-coated) columns, whereby the $1 \mathrm{mSL}$ particle experiences the greatest retention, and the smaller ENPs exhibit lower attachment efficiencies than $1 \mathrm{mSL}$. It is of interest to point out that the Qvalue for the $1 \mathrm{mSL}$ particle exceeds unity $\left({ }^{\prime}\right)$ which may be an indication that physical straining contributes to the retention of this particle in the biofilm-coated sand column.

The average $\zeta$-potential of the biofilm-coated sand is less negative than that of the clean sand, suggesting that the negatively charged ENPs should experience lessened EDL repulsion upon approach to the biofilm coating. Overall, the particles do show higher retention onto sand coated with a mature

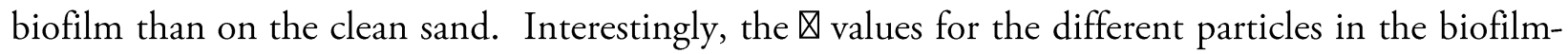
coated sand packed column are not dramatically high; only the larger $1 \mathrm{mSL}$ particle exhibits an attachment efficiency greater than unity. 
EPS are important components of biofilms ${ }^{45}$ and thus it is of interest to understand the role of EPS in defining the retention properties of biofilm. When the clean sand surface is coated with EPS extracted from $P$. aeruginos $a$ biofilms, the larger $1 \mathrm{mSL}$ particle experiences similar high retention as in the biofilm-coated column. Although the $\zeta$-potential of the EPS-coated sand is the same as that of the biofilm-coated sand (Figure 1), the attachment efficiencies of the three ENPs are different in the two systems (Figure 3). Specifically, the $\nabla$ values of the ENPs in columns packed with EPS-coated sand are lower than those observed in the biofilm-coated system. These results demonstrate that the electrokinetic properties of these complex collector surfaces are not necessarily good predictors of particle retention potential. Figure 3 also shows that even though EPS is considered to make up an important fraction of the biofilm matrix ${ }^{14,36}$, EPS coatings are not necessarily good surrogates for biofilms.

The selection of ENPs that are similar in size but different in surface coating allows us to examine the influence of surface chemistry on ENP-surface interactions in the different experimental systems. The $20 n \mathrm{SL}$ and $20 n \mathrm{CL}$ have comparable $\bigotimes$ values in the clean sand columns; however, their deposition behavior in the coated-sand columns is quite different. The attachment efficiency of the sulfonated nanoparticle is similar in the clean, LB-, and EPS-coated sand, whereas the deposition of the carboxylated nanoparticle is considerably lower in the LB-coated sand (when compared to clean sand) (Figure 3). The retention of the QD is also much lower in the LB-coated and EPS-coated sand columns (compared to clean sand). In contrast, Tong et al. ${ }^{5}$ observed increased retention of $n \mathrm{C}_{60}$ particles on silica surfaces coated with EPS extracted from E. coli (when compared to clean silica). These observations illustrate the importance of the ENP surface chemistry in influencing the 
nanoparticle's interaction with EPS coatings or other conditioning films. Likewise, we note that the like-sized 20nSL and 20nCL particles exhibit different affinities for the biofilm-coated sand, whereas their transport behavior is similar in the clean sand column. Although the coatings on the particles used here are fairly well defined and uniform, the compositions of the collector surface coatings (e.g., LB and biofilm) are highly complex. Hence, it is not straightforward to identify the specific interactions that may favor or disfavor the attachment of the sulfonated versus carboxylated nanoparticles onto these collector coatings. The architecture of the coated collector surfaces is another parameter that can influence the retention of particles in the granular matrix and that can vary from one type of coating to another. Thus, future studies aimed at understanding the role of such collector coatings on particle transport and fate should also consider comprehensive chemical and physical characterization of the coated surfaces. The composition of biofilm, EPS and other conditioning films that may be present in natural or engineered aquatic environments is highly variable ${ }^{36}$, further complicating our ability to generalize the potential influence of these coatings on ENP transport and fate.

\section{Environmental Implications}

An understanding of ENP fate in water-saturated granular porous media is needed to establish the potential risks of these materials to aquatic and soil environments as well as to public health. Although the number of studies examining the transport, aggregation, retention and transformation of various ENPs in aquatic systems is growing rapidly ${ }^{3}$, our knowledge on ENP-biofilm interactions is very limited. This and other studies, 7-9 demonstrate how the presence of biofilm grown on collector 
surfaces can result in increased retention of ENPs in natural and engineered granular aquatic environments. Yet, Leon-Morales et al. ${ }^{9}$ also reported that changes in water chemistry can result in increased mobility of a nanoparticle (laponite) in biofilm-coated sand. Hence, the influence of biofilm on ENP transport and fate is not clear. In this study, the use of ENPs that are similar in size but having different surface functionalities demonstrate how particle surface chemistry can influence ENP interactions with biofilm-, EPS, or other film-coated environmental surfaces. Experiments conducted with columns packed with clean sand or sand that had been pre-coated with growth medium, biofilm or bacterial EPS reveal that ENPs can interact differently with a mature biofilm versus a conditioning film.

Evidently, particle transport in a biofilm-coated matrix is a complex phenomenon which cannot be completely described by any single process. While straining and deposition in the secondary energy minimum appear to play a more important role for the larger particle $(1 \mathrm{mSL})$, physicochemical or specific interactions and/or steric repulsion between the particles and various collector surfaces also likely contribute to the overall particle and ENP transport behavior. Thus, the electrokinetic properties of the particle/ENP and collector surfaces are not necessarily good predictors of transport behavior in a biofilm- or polymer-coated granular matrix.

The experiments presented here were performed with selected few ENPs in columns packed with uniformly-sized quartz sand and biofilms comprising of only one strain of $P$. aeruginosa. However, natural soils and engineered granular filtration systems contain heterogeneous biofilms of mixed organisms, coating grains of variable shape, size, and surface chemistry. Ongoing research in our laboratory aims at extending this work to examine the transport behavior of ENPs having different 
chemistries in biofilm-laden soils over a broader range of environmentally relevant conditions. An improved understanding of ENP transport in natural and engineered granular aquatic environments will lead to recommendations for safer disposal practices of ENP-laden wastewaters and better predictions of ENP contamination potential in groundwater. 


\section{Acknowledgements}

This research was supported by NSERC, FQRNT, the CRC Program and the CFI. We acknowledge the contributions of C. O'May, H. A. Lam, and M. Renker for technical support in setting up the experimental system and protocols and I. R. Quevedo for assistance with DLVO calculations and TEM imaging. 


\section{Supporting Information Available}

Additional characterization of selected particles, values of parameters used in calculation of particle attachment efficiencies, and representative breakthrough curves for tracer and $1 \mathrm{mSL}$ particle in columns packed with clean sand or sand coated with biofilm, EPS, or growth medium. This information is available free of charge via the Internet at http://pubs.acs.org/. 


\section{References}

1. Roco, M. C., Broader Societal Issues of Nanotechnology. J. Nanopart. Res. 2003, 5, 181-189.

2. Biswas, P.; Wu, C. Y., Nanotechnology and the Environment. J. Air \& Waste Manage. Assoc. 2005, 55, 708-746.

3. Petosa, A. R.; Jaisi, D. P.; Quevedo, I. R.; Elimelech, M.; Tufenkji, N., Aggregation and deposition of engineered nanomaterials in aquatic environments: role of physicochemical interactions. Environmental Science and Technology 2010, 44, (17), 6532-49.

4. Tufenkji, N.; Ryan, J. N.; Elimelech, M., The promise of bank filtration. Environmental Science \& Technology 2002, 36, 422a-428a.

5. Tong, M.; Ding, J.; Shen, Y.; Zhu, P., Influence of biofilm on the transport of fullerene (C60) nanoparticles in porous media. Water Research 2010, 44, (4), 1094-103.

6. Peulen, T. O.; Wilkinson, K. J., Diffusion of nanoparticles in a biofilm. Environmental Science \& Technology 2011, 45, (8), 3367-73.

7. Leon-Morales, C. F.; Leis, A. P.; Strathmann, M.; Flemming, H. C., Interactions between laponite and microbial biofilms in porous media: implications for colloid transport and biofilm stability. Water Research 2004, 38, (16), 3614-26.

8. Habimana, O.; Steenkeste, K.; Fontaine-Aupart, M. P.; Bellon-Fontaine, M. N.; Kulakauskas, S.; Briandet, R., Diffusion of nanoparticles in biofilms is altered by bacterial cell wall hydrophobicity. Applied and Environmental Microbiology 2011, 77, (1), 367-8.

9. Leon Morales, C. F.; Strathmann, M.; Flemming, H. C., Influence of biofilms on the movement of colloids in porous media. Implications for colloid facilitated transport in subsurface environments. Water Research 2007, 41, (10), 2059-2068.

10. Hall-Stoodley, L.; Costerton, J. W.; Stoodley, P., Bacterial biofilms: from the natural environment to infectious diseases. Nature reviews. Microbiology 2004, 2, (2), 95-108.

11. Hunter, R. J., Foundaations of Colloid Science. Oxford University Press: New York, 2001.

12. Ohshima, H., A simple expression for Henry's function for the retardation effect in electrophoresis of spherical colloidal particles. Journal of Colloid and Interface Science 1994, 168, 269-271.

13. Schinner, T.; Letzner, A.; Liedtke, S.; Castro, F. D.; Eydelnant, I. A.; Tufenkji, N., Migration behavior of selected bacterial pathogens in agricultural soil and quartz sand. Water Research 2010, 44, 1182-1192.

14. Wingender, J.; Strathmann, M.; Rode, A.; Leis, A.; Flemming, H. C., Isolation and biochemical characterization of extracellular polymeric substances from Pseudomonas aeruginosa. Methods Enzymol 2001, 336, 302-14.

15. Gomez-Suarez, C.; Pasma, J.; van der Borden, A. J.; Wingender, J.; Flemming, H. C.; Busscher, H. J.; van der Mei, H. C., Influence of extracellular polymeric substances on deposition and redeposition of Pseudomonas aeruginosa to surfaces. Microbiology 2002, 148, (4), 1161-9.

16. Liu, H.; Fang, H. H., Extraction of extracellular polymeric substances (EPS) of sludges. Journal of Biotechnology 2002, 95, (3), 249-56.

17. Truesdail, S. E.; Westermann-Clark, G. B.; Shah, D. O., Apparatus for streaming potential measurements on granular filter media. Journal of Environmental Engineering 1998, 124, (12), 1228-1232.

18. Fairbrother, F.; Mastin, H., Studies in electro-endosmosis Part I. Journal of the Chemical Society 1924, $125,2319-2330$.

19. Domingos, R. F.; Baalousha, M. A.; Ju-Nam, Y.; Reid, M. M.; Tufenkji, N.; Lead, J. R.; Leppard, G. G.; Wilkinson, K. J., Characterizing manufactured nanoparticles in the environment: multimethod determination of particle sizes. Environmental Science and Technology 2009, 43, (19), 7277-84. 
20. Chen, K. L.; Elimelech, M., Aggregation and deposition kinetics of fullerene (C60) nanoparticles. Langmuir 2006, 22, 10994-11001.

21. Quevedo, I. R.; Tufenkji, N., Influence of solution chemistry on the deposition and detachment kinetics of a CdTe quantum dot examined using a quartz crystal microbalance. Environmental Science and Technology 2009, 43, (9), 3176-82.

22. Chen, K. L.; Smith, B. A.; Ball, W. P.; Fairbrother, D. H., Assessing the colloidal properties of engineered nanoparticles in water: case studies from fullerene C-60 nanoparticles and carbon nanotubes. Environmental Chemistry 2010, 7, (1), 10-27.

23. Fatisson, J.; Domingos, R. F.; Wilkinson, K. J.; Tufenkji, N., Deposition of TiO2 Nanoparticles onto Silica Measured Using a Quartz Crystal Microbalance with Dissipation Monitoring. Langmuir 2009, 25, (11), 6062-6069.

24. Pelley, A. J.; Tufenkji, N., Effect of particle size and natural organic matter on the migration of nanoand microscale latex particles in saturated porous media. J Colloid Interface Sci 2008, 321, (1), 74-83.

25. Wanner, O.; Cunningham, A. B.; Lundman, R., Modeling biofilm accumulation and mass transport in a porous medium under high substrate loading. Biotechnology and Bioengineering 1995, 47, (6), 703-712.

26. Tufenkji, N.; Elimelech, M., Correlation equation for predicting single-collector efficiency in physicochemical filtration in saturated porous media. Environmental Science and Technology 2004, 38, (2), 52936.

27. Verwey, E. J. W.; Overbeek, J. T. G., Theory of Stability of Lyophobic Colloids. Elsevier Amsterdam, 1948.

28. Derjaguin, B. V.; Landau, L. D., Theory of the Stability of strongly charged particles in solutions of electrolytes Acata Physicochim. URSS 1941, 14, 733-762.

29. Gregory, J., Interaction of unequal double layers at constant charge. Journal of Colloid and Interface Science 1975, 51, (1), 44-51.

30. Gregory, J., Approximate expressions for retarded van der Waals interaction. Journal of Colloid and Interface Science 1981, 83, (1), 138-45.

31. Hahn, M. W.; Abadzic, D.; O'Melia, C. R., Aquasols: On the Role of Secondary Minima. Environmental Science \& Technology 2004, 38, 5915-5924.

32. Kuznar, Z. A.; Elimelech, M., Direct microscopic observation of particle deposition in porous media: Role of the secondary energy minimum Colloids and Surfaces A: Physicochem. Eng. Aspects 2007, 294, 156-162. 33. Tufenkji, N.; Miller, G. F.; Ryan, J. N.; Harvey, R. W.; Elimelech, M., Transport of Cryptosporidium oocysts in porous media: Role of straining and physicochemical filtration. Environmental Science \& Technology 2004, 38, 5932-5938.

34. Bradford, S. A.; Bettahar, M.; Simunek, J.; Genuchten, M. T. V., Straining and attachment of colloids in physically heterogeneous porous media Vadose Zone Journal 2004, 3, 384-394.

35. Bos, R.; Van Der Mei, H. C.; Busscher, H. J., Physico-chemistry of initial microbial adhesive interactions - Its mechanisms and methods for study. FEMS Microbiology Reviews 1999, 23, (2), 179-229.

36. Flemming, H. C.; Wingender, J., Relevance of microbial extracellular polymeric substances (EPSs) Part I: Structural and ecological aspects. In Water Science and Technology, 2001; Vol. 43, pp 1-8.

37. Jiang, X.; Tong, M.; Li, H.; Li, H., Deposition kinetics of zinc oxide nanoparticles on natural organic matter coated silica surfaces. Journal of Colloid and Interface Science 2010, 350, (2), 427-434.

38. Chen, K. L.; Elimelech, M., Interaction of Fullerene (C60) Nanoparticles with Humic Acid and Alginate Coated Silica Surfaces: Measurements, Mechanisms, and Environmental Implications. Environmental Science \& Technology 2008, 42, 7607-7614.

39. Eydelnant, I. A.; Tufenkji, N., Cranberry derived proanthocyanidins reduce bacterial adhesion to selected biomaterials. Langmuir 2008, 24, (18), 10273-10281. 
40. Napper, D. H., Steric Stabilization. Journal of Colloid and Interface Science 1977, 58, (2), 390-407.

41. Rijnaarts, H. H. M.; Norde, W.; Lyklema, J.; Zehnder, A. J. B., The Isoelectric Point of Bacteria as an Indicator for the Presence of Cell-Surface Polymers That Inhibit Adhesion. Colloids and Surfaces B-Biointerfaces 1995, 4, (4), 191-197.

42. Tufenkji, N.; Dixon, D. R.; Considine, R.; Drummond, C. J., Multi-scale Cryptosporidium/sand interactions in water treatment. Water Research 2006, 40, (18), 3315-3331.

43. Liu, Y.; Yang, C. H.; Li, J., Adhesion and retention of a bacterial phytopathogen Erwinia chrysanthemi in biofilm-coated porous media. Environmental Science and Technology 2008, 42, (1), 159-165.

44. Drury, W.; Stewart, P.; Characklis, W. G., Transport of 1-mm latex particles in Pseudomonas aeruginosa biofilms. . Biotechnology and Bioengineering 1993, 42, 111-117.

45. Strathmann, M.; Leon-Morales, C. F.; Flemming, H. C., Influence of Biofilms on Colloid Mobility in the Subsurface. In Colloidal Transport in Porous Media, Frimmel, F. H.; von der Kammer, F.; Flemming, H. C., Eds. Springer: Berlin Heidelberg, 2007; pp 143-173.

46. Langmark, J.; Storey, M. V.; Ashbolt, N. J.; Sentrom, T.-A., Accumulation and fate of microorganisms and microspheres in biofilms formed in a pilot-scale water distribution system. Applied and Environmental Microbiology 2005, 71, 706-712.

47. Okabe, S.; Kuroda, H.; Watanabe, Y., Uptake and release if inert fluorescence particles by mixed population biofilms. Biotechnology and Bioengineering 1997, 53, 459-469. 
Table 1. Selected nano- and micron-sized particles used in transport experiments.

\begin{tabular}{ccccccc}
\hline Particle & $\begin{array}{c}\text { Core } \\
\text { Material }\end{array}$ & $\begin{array}{c}\text { Surface } \\
\text { Functional } \\
\text { Groups }\end{array}$ & $\begin{array}{c}\text { Reported } \\
\text { Particle } \\
\text { Diameter } \\
(\mathbf{n m})\end{array}$ & $\begin{array}{c}\text { Measured } \\
\text { Diameter } \\
(\mathbf{n m})\end{array}$ & $\begin{array}{c}\text { EPM } \\
(\mu \mathbf{m} \mathbf{~ c m} / \mathbf{V} \text { sec })\end{array}$ & $\begin{array}{c}\text { Zeta } \\
\text { Potential } \\
(\mathbf{m V})\end{array}$ \\
\hline $1 \mathrm{mSL}$ & $\begin{array}{c}\text { polystyrene } \\
\text { latex }\end{array}$ & sulfate & 1000 & $925 \pm 80$ & $-5.5 \pm 0.1$ & $-65.1 \pm 3.8$ \\
$20 n \mathrm{SL}$ & $\begin{array}{c}\text { polystyrene } \\
\text { latex }\end{array}$ & sulfate & 20 & $33 \pm 4$ & $-2.7 \pm 0.5$ & $-45.1 \pm 6.7$ \\
$20 n \mathrm{CL}$ & $\begin{array}{c}\text { polystyrene } \\
\text { latex }\end{array}$ & carboxyl & 20 & $26 \pm 3$ & $-2.3 \pm 0.8$ & $-41.0 \pm 9.3$ \\
$\mathrm{QD}$ & CdSe/ZnS & carboxyl & 10 & $108 \pm 22$ & $-2.2 \pm 0.4$ & $-19.5 \pm 2.8$ \\
\hline
\end{tabular}

Table 2. Calculated DLVO interaction energy parameters $\left(\boldsymbol{\Phi}_{\max }\right.$ and $\boldsymbol{\Phi}_{2^{\circ} \text { min }}$ ) reported in units of $k T$ for the different particle-sand systems ${ }^{\mathrm{a}}$.

\begin{tabular}{ccccc} 
Sand & \multicolumn{5}{c}{ Selected Particle } \\
Treatment & 1 $\mathbf{m S L}$ & 20nSL & 20nCL & QD \\
\hline \multirow{2}{*}{ clean sand } & 177.7 & 4.5 & 2.9 & 5.5 \\
& 1.2 & 0.1 & 0.1 & 0.3 \\
\hline
\end{tabular}

${ }^{\mathrm{a}}$ Values of $\Phi_{\max }$ are shown in regular font and values of $\Phi_{2^{\circ} \text { min }}$ are shown in italics. 


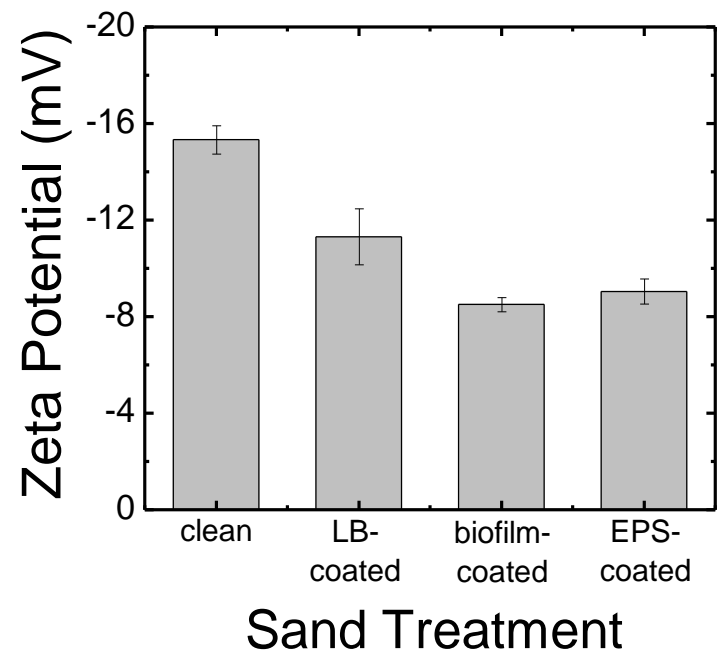

Figure 1. Zeta potential of the collector (sand) surfaces used in this study. Error bars represent 95\% confidence intervals. 

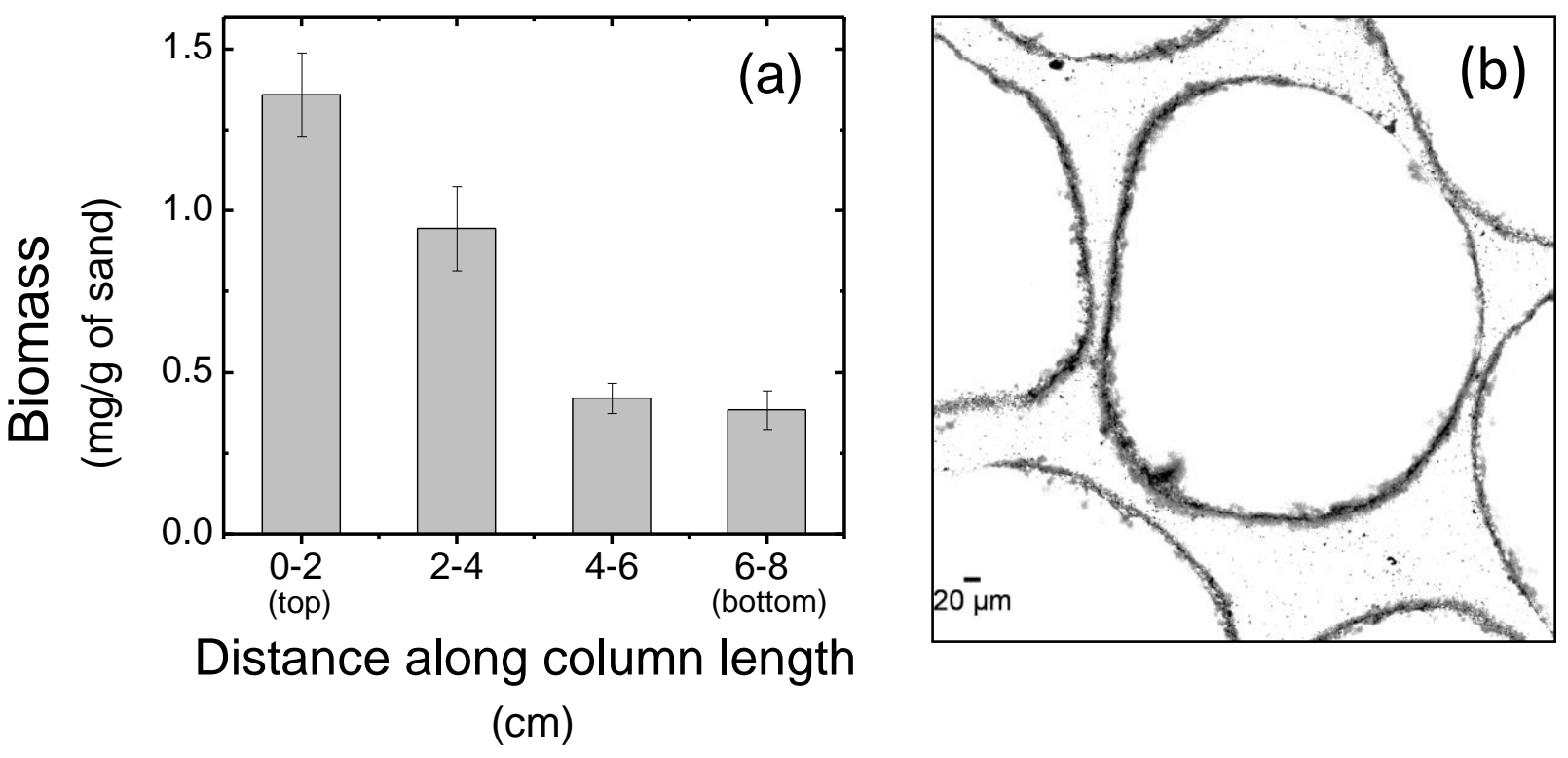

Figure 2. Characterization of biofilm-coated sand in the packed column. (a) Total biomass as a function of bed depth; (b) Biofilm coating of sand grains as observed by CLSM at 10× magnification (image has been converted to black and white where the biomass is shown in black). 


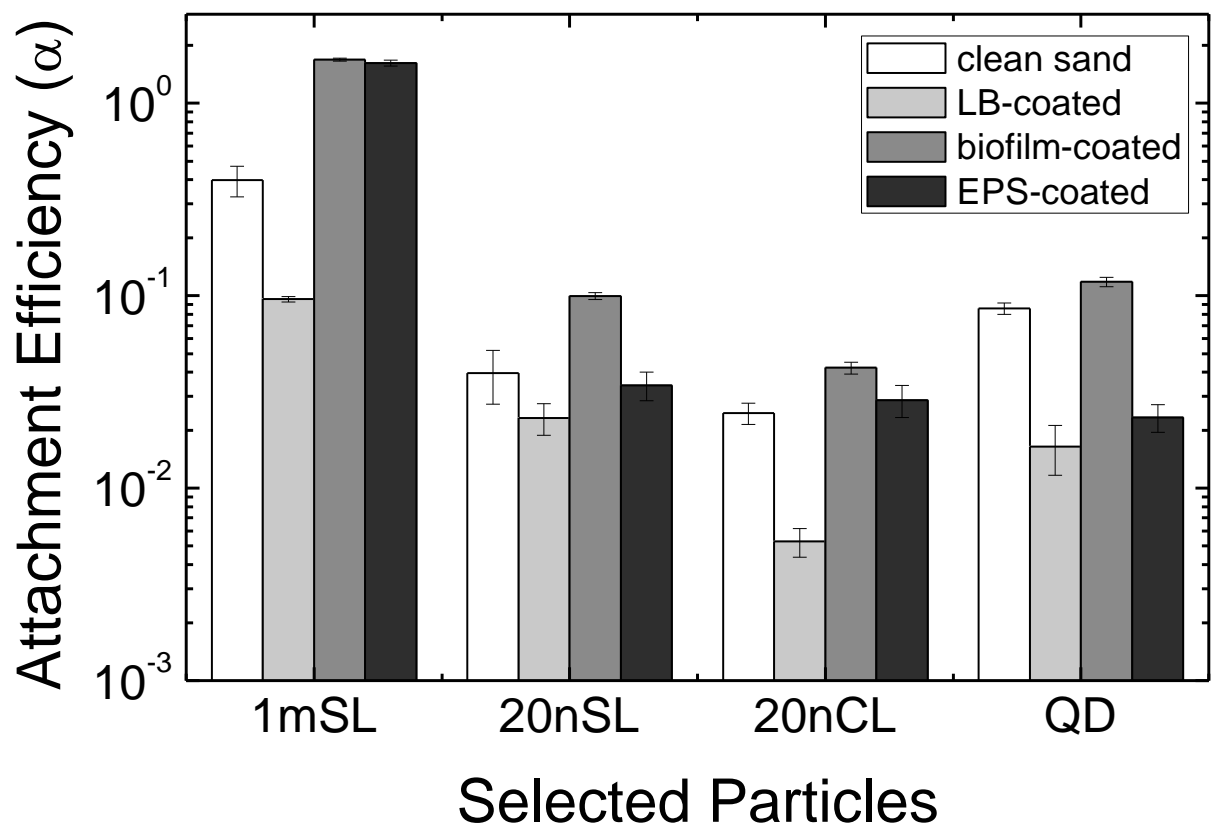

Figure 3. Attachment efficiencies of a microsphere and three selected ENPs on clean and coated sand surfaces. Sand coatings include a conditioning film of diluted LB medium (LB-coated), EPS extracted from $P$. aeruginosa biofilm (EPS-coated), and $P$. aeruginosa biofilm (biofilm-coated). 
TOC Artwork

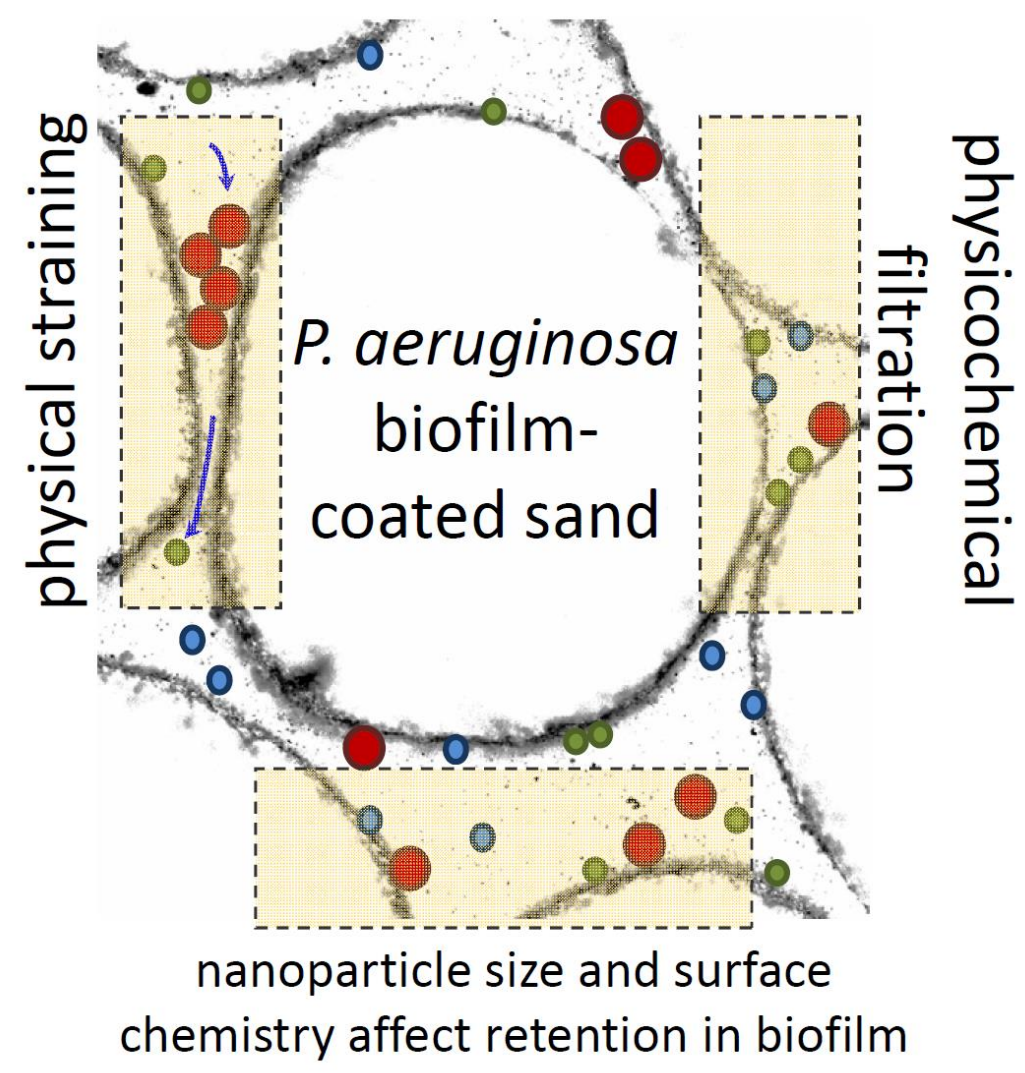

